\title{
MOLECULAR ENGINEERING OF AN ORTHOPAEDIC IMPLANT: FROM BENCH TO BEDSIDE
}

\author{
I.M. Shapiro ${ }^{1, *}$, N.J. Hickok ${ }^{1}$ J. Parvizi ${ }^{2}$, S. Stewart ${ }^{3}$ and T.P. Schaer ${ }^{3}$ \\ ${ }^{1}$ Department of Orthopaedic Surgery, Thomas Jefferson University, Philadelphia, PA, USA \\ ${ }^{2}$ Department of Orthopaedic Surgery and the Rothman Institute, Thomas Jefferson University, \\ Philadelphia, PA, USA, \\ ${ }^{3}$ Comparative Orthopaedic Research Laboratory, Department of Clinical Studies, New Bolton Center, \\ University of Pennsylvania School of Veterinary Medicine, Kennett Square, PA, USA
}

\begin{abstract}
The use of metallic implants has revolutionised the practice of orthopaedic surgery. While the safety and biocompatibility of these devices are excellent, a small percentage becomes infected. These infections are due to the formation of a biofilm that harbours bacteria encased in a complex extracellular matrix. The matrix serves as a barrier to immune surveillance as well as limiting the biocidal effects of systemic and local antibiotics. The objective of the review is to describe a novel approach to controlling implant infection using an antibiotic that is linked to titanium through a self-assembled monolayer of siloxy amines. We show that the hybrid-engineered surface is stable, biocompatible and resists colonisation by bacterial species most commonly associated with implant-related infections. Studies with rodent bone infection models suggest that the engineered titanium surface prevents bone infection. Results of a very recent investigation utilising a sheep model of infection indicate that the titanium-tethered antibiotic controls infection without compromising bone formation and remodelling. From all of these perspectives, the tethered antibiotic holds promise of providing a novel and practical approach to reducing implant-associated infections.
\end{abstract}

Keywords: vancomycin; orthopaedic implant; biofilm; infection; silane chemistry; animal model

*Address for correspondence:

Irving M. Shapiro

Department of Orthopaedic Surgery,

Jefferson Medical College,

Thomas Jefferson University,

1015 Walnut Street, Suite 510,

Philadelphia, PA 19146, USA

Telephone Number: 001-215-955-7217

E-mail: irving.shapiro@jefferson.edu

\section{The need for implant tethered antibiotics}

Orthopaedic surgery frequently requires the placement of a foreign body, such as a prosthetic joint or hardware, deep within the appendicular or axial skeleton. The majority of these implants reside within these tissues infectionfree; a small percentage, however, become infected. The incidence of infection (Periprosthetic Joint Infection, PJI) following total joint arthroplasty, ranges from $1 \%$ to $4 \%$ for primary total knee arthroplasty and $1 \%$ to 2 $\%$ for primary total hip arthroplasty (Cierny et al., 2002; McPherson et al., 2002; Geipel and Herrmann, 2004). These infections are thought to be due to the formation of a biofilm on the surface of the implant. The biofilms harbour bacteria encased in a complex extracellular matrix that serves as a barrier to immune surveillance and limits the efficacy of systemic and local antibiotics (Stewart and Costerton, 2001; Bahar, 2002; Donlan et al., 2002). Once established, these infections can rarely be eradicated by the host defence or by administration of antimicrobial agents. Typically, revision surgeries necessitate not only removal of the biofilm-contaminated implant, but also bone debridement and prolonged antimicrobial treatment (Toms et al., 2006). These procedures are costly and invariably lead to further pain, distress and suffering.

Because the implant is an ideal surface for biofilm formation, a number of approaches have been developed to prevent or disrupt its development. The one chosen will depend on the nature of the surgical problem, the type of implant utilised, the type of invading organisms and the health and age of the patient. In most instances however, the implant must be first removed, the infected tissue debrided and an antibiotic eluting spacer positioned in the bone prior to subsequent re-insertion of the implant. Not surprisingly, a considerable number of agents have been used to deliver antibiotics to the infected bone. Foremost among these is the targeted delivery of antimicrobial agents in polymethylmethacrylate cement (Hanssen, 2004), and beads (Moojen et al., 2008), calcium sulphate (Grimsrud et al., 2011), calcium phosphate (Watts et al., 2011) and very recently a resorbable bioactive silica calcium phosphate nanocomposite (El-Ghannam et al., 2010). While each of these materials is of obvious clinical value, immediate high levels of release can complicate healing and slow implant osseointegration. More importantly, when the level of the eluted antibiotic is lower than the minimal inhibitory concentration, conditions are ideal for the emergence of resistant strains (Costerton, 2005). Finally, the antibiotic-depleted carrier or remnants of the carrier 
can serve as a nidus for promoting bacterial targeting and adherence, thereby contributing to biofilm formation.

One approach to overcoming the problems mentioned above is to covalently bond a biocidal molecule to the surface of an implant. For titanium, linkages reported include diphosphonic acid (Danahy et al., 2004), plasma animation (Puleo et al., 2002), silanisation (Wojcik and Puleo, 1997; Antoci et al., 2007d; Sechi et al., 2007), photopolymerisation (Lawson et al., 2007), p-nitrophenyl chloroformate treatment (Mikulec and Puleo, 1996) and PEGylation (Khoo et al., 2009). This type of surface would prevent bacterial attachment and therefore block biofilm formation. Our on-going studies have shown that an antibiotic, vancomycin (VAN), can be linked to titanium through a self-assembled monolayer (SAM) of siloxy amines. Moreover, the hybrid engineered surfaces resist colonisation by bacterial species that are most commonly associated with implant-related infections.

The goal of this review is to briefly describe the chemistry of attachment, and the antibacterial properties of the tethered antibiotic. We discuss the import of such a surface in terms of bacterial attachment and colonisation, biofilm formation and antibiotic stability and activity (Antoci et al., 2007a,b, 2008; Ketonis et al., 2009; Edupuganti et al., 2007). Finally, we describe experiments in which the modified implant is used to prevent infection and permit bone repair to take place in a large animal model. The results of the investigation lend strong support to the notion that antibiotic modification of orthopaedic hardware promotes bone healing in the presence of significant bacterial contamination. From this perspective, the tethered bioactive molecules hold promise of providing a new and practical approach to reducing implant-associated infections.

\section{Synthesis of a tethered antibiotic surface}

The starting material for the studies was either titanium alloy (Ti6Al4V) or commercially pure titanium (cpTi) - the most commonly used metals in orthopaedic implants. The antibiotic chosen for both the in vitro and in vivo studies was VAN. Clinical reasons for choosing this antibiotic have been provided in studies reported elsewhere (Fulkerson et al., 2005); these include: 1 . the VAN site of action is within the bacterial cell wall, and hence freely accessible to the antibiotic, 2. the mechanism of action of VAN is reversible, suggesting that the surface would retain its biocidal properties after interaction with cell wall constituents (Loll and Axelsen, 2000; Hickok et al., 2011).

The antibiotic was attached covalently to the metal by first oxidising the titanium surface and then coupling an organic molecule, aminopropyltriethoxysilane (APTES) to the oxide layer. This agent provided a terminal reactive amine groups for attachment of linker and antibiotic molecules. The purpose of the linker molecules is to extend the VAN from the titanium surface. It was presumed that the flexible hydrophilic linkers would enhance antibiotic entry into the bacterial cell wall. Methodologies for tethering VAN to titanium alloy via silane linkages have been described elsewhere (see Edupuganti et al., 2007). For the studies to be described towards the end of this review, cpTi was stripped of the anodised surface layer in a nitrohydrofluoric acid bath. It was then oxidised (passivated) by hydrothermal aging (Ketonis et al., 2009) and an aminopropyl self-assembled monolayer (SAM) formed by reaction with APTES. Following cross-linking of the SAM and washing to remove excess APTES, two Fmoc-aminoethoxyethoxy-acetate (Fmoc-AEEA) linkers were sequentially coupled to the free amino group after activation with $\mathrm{O}$-(7-azabenzo-triazole-1-yl)-1,1,3,3tetramethyluronium hexafluorophosphate (HATU) in the presence of di-isopropylethylamine; the Fmoc protecting group was then removed by reaction with piperidine. The linker (AEEA-AEEA-APTES) was then coupled with a fourfold molar excess of VAN, using HATU chemistry as described above (Jose et al., 2005). We predict that the linkers provide a 3-4 $\mathrm{nm}$ arm length extension from the metal surface; this arm provides the freedom needed for the antibiotic to interact with bacterial cell wall constituents (Edugupanti et al., 2007). The VAN binds to Lys-D-AlaD-Ala termini of peptidoglycan precursors in the bacterial cell wall and interferes with macromolecular synthesis (Kahne et al., 2005).

Two approaches were utilised to confirm that we had generated a VAN-AEEA-AEEA-APTS-titanium surface structure. First, the surface was evaluated by MALDI-TOF fragmentation analysis which revealed the presence of $\mathrm{H}_{2} \mathrm{~N}-\mathrm{VAN}$, AEEA-VAN as well as APTS-AEEA-AEEAVAN (Jose et al., 2005). The second approach was to monitor the addition of each of the major moieties onto the titanium surface: fluorescamine was used to label the generation of free primary amines while the ninhydrin assay, a colorimetric assay which monitors colour adducts was used to measure free primary amines (Jose et al., 2005; Antoci et al., 2007c). Finally, we used antibodies raised against the antibiotic to quantify VAN coverage (see below). Results of these studies revealed that there is close to the theoretical maximum of 6 APTS, linkers $/ \mathrm{nm}^{2}$ (Lee et al., 2005), and that linker and antibiotic addition on the titanium surface proceeds stoichiometrically at high efficiency (Edugupanti et al., 2007). Moreover, based on the titanium area, we calculated that each bacterium would be exposed to at least 100,000 molecules of antibiotic. This high surface density of antibiotic is important not just in terms of providing a coherent antibiotic barrier that would mitigate biofilm formation, but it would also serve to prevent development of resistance (Antoci et al., 2008). Before completing this section of the review it should be acknowledged that bonding VAN to an aminopropylated surface by solid phase peptide synthesis is a very useful general chemical procedure. We have used it to bond a number of antibiotics to metals and the technology can be readily applied to other bioactive molecules (Chen and Wickstrom, 2010; Ketonis et al., 2010).

\section{Stability and antimicrobial activity of the vancomycin tethered implant surface}

Chemical and physical evaluation techniques indicated that the synthetic reactions generated a homogeneous layer of tethered antibiotic. Since the chemical syntheses utilised a number of organic solvents that could denature 


\section{Control}
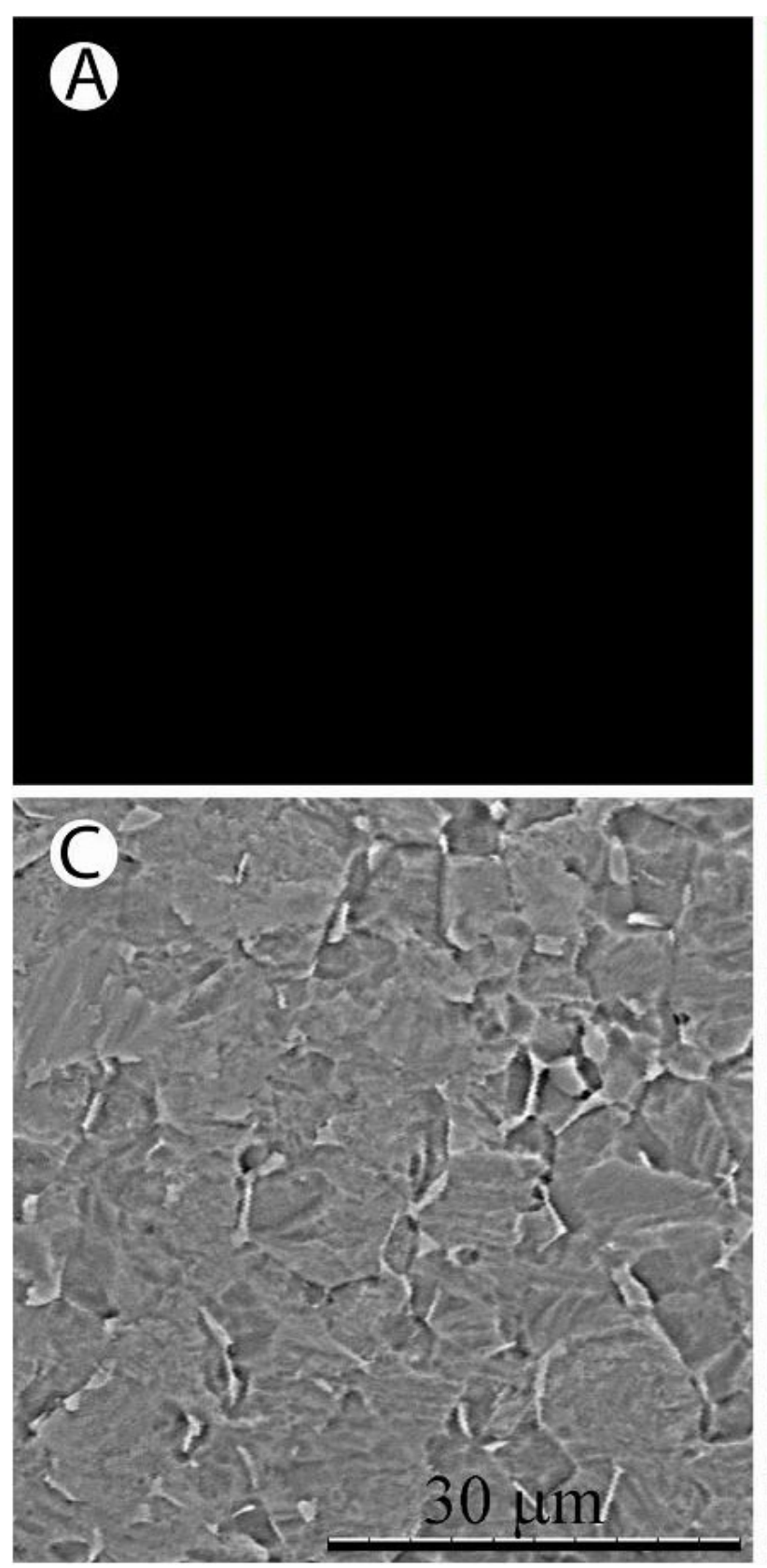

VAN-Ti
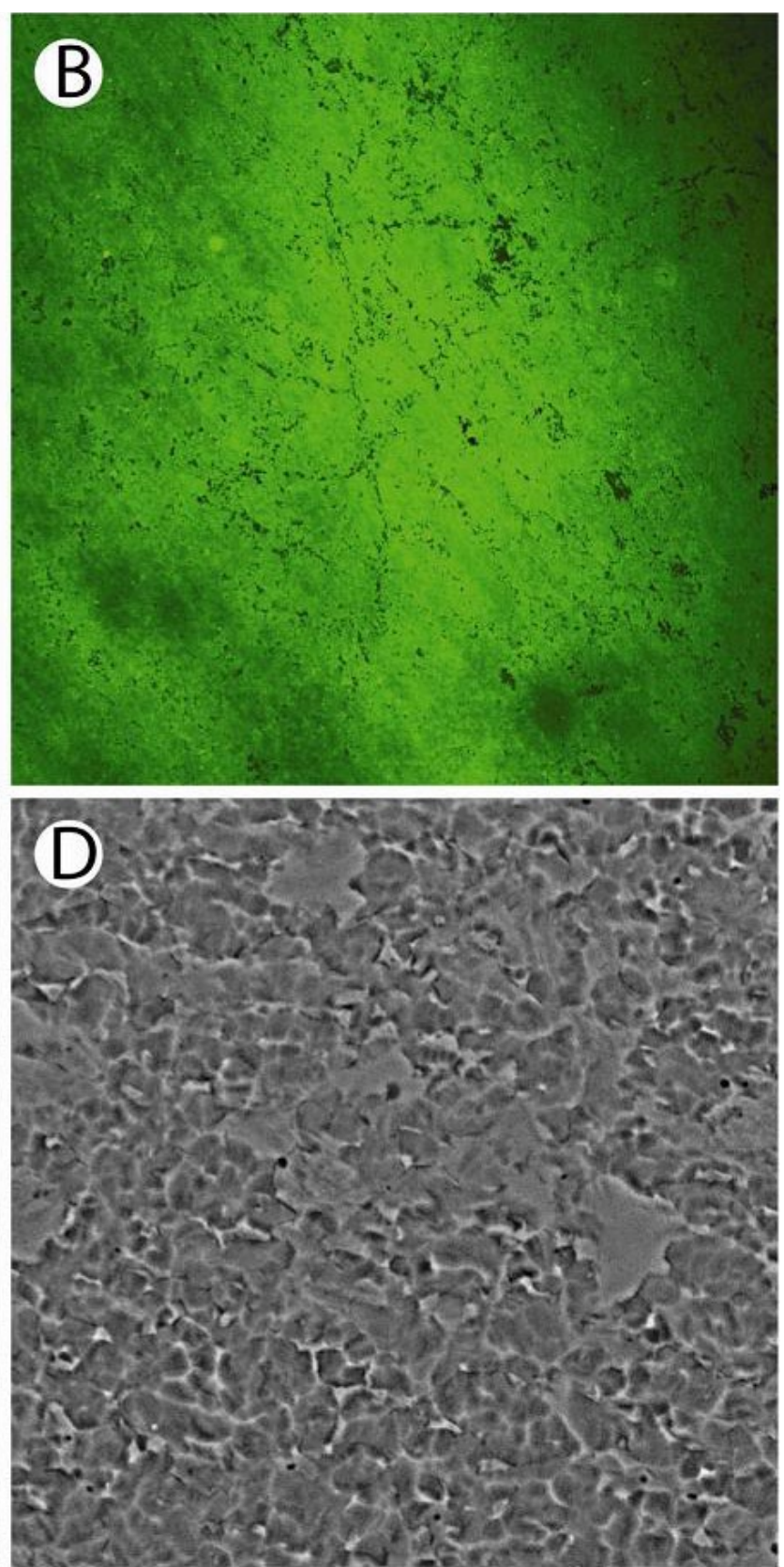

Fig. 1. Characterisation of the vancomycin (VAN-Ti) tethered surfaces. (A) and (B) Distribution of vancomycin on a titanium surface. (A) Control surface in the absence of vancomycin. (B) Vancomycin coupled titanium surface. The surface was treated with an antibody to vancomycin and imaged using confocal microscopy. Note the uniform distribution of the antibiotic over the surface of the metal. (C) and (D) SEM of control and antibiotic treated surface. (C) Untreated control surface. The grain boundaries are clearly visible. (D) Vancomycin treated titanium surface. Again, the grain boundaries are visible and possibly of smaller diameter than the untreated titanium surface. Scale bar $=30 \mu \mathrm{m}$.

the antibiotic, we needed to confirm that these agents together with the large number of washing steps did not decrease the stability and antimicrobial activity of the tethered VAN. Related to these procedures, it was also important to confirm that the synthetic reactions did not etch or otherwise modify the titanium surface structure. The presence of the surface bound antibiotic was determined using an antibody against VAN and imaged by confocal laser microscopy. Fig. 1A,B shows that the VAN was distributed uniformly over the surface of the titanium, while scanning electron microscopy indicated that changes in surface structure of the metal were minimal.

Recognising that the tethered antibiotic would be exposed to a number of different aqueous environments both prior to and during implantation, it was necessary to evaluate its fluid stability and activity. To gain some insight 

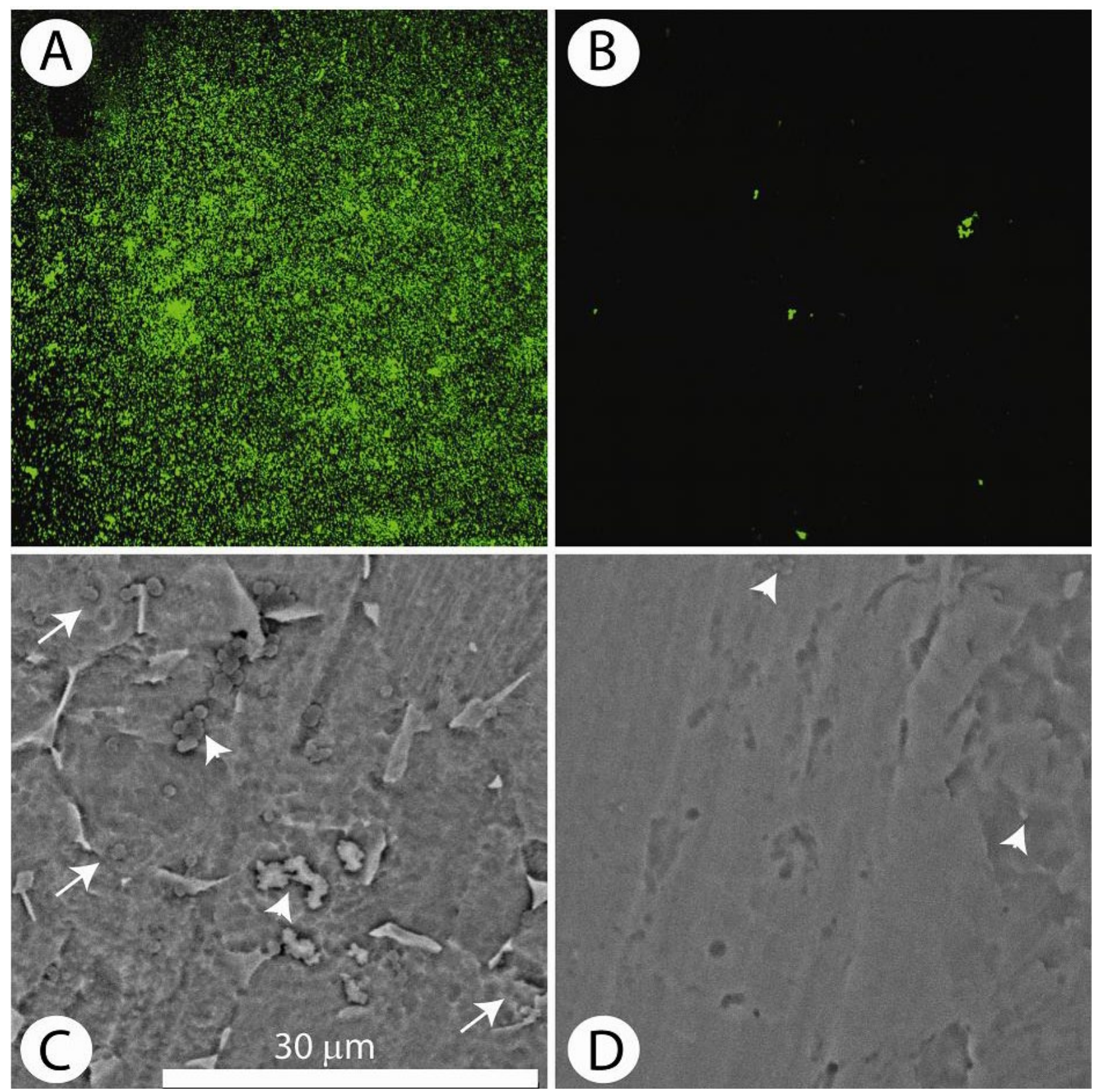

Fig. 2. S. aureus accumulation on the vancomycin (VAN-Ti) tethered surface. (A) Distribution of bacteria $\left(10^{3} \mathrm{cfu} / \mathrm{mL}\right.$, $24 \mathrm{~h}$ ) stained with the Live/Dead assay on the control surface (no antibiotic) and visualised by confocal microscopy. The high green fluorescence indicates extensive bacterial colonisation. (B) Distribution of bacteria on the tethered antibiotic surface (VAN-Ti) stained with the Live/Dead assay. Note the absence of green fluorescence indicating minimal adherent bacteria. (C) and (D) SEM of control and treated titanium surfaces. (C) SEM of the control surface following incubation with bacteria for $24 \mathrm{~h}$. Arrowheads show the presence of small colonies of bacteria; arrows indicate biofilm encased bacteria. (D) Vancomycin treated titanium surface. Note the few bacteria on the treated surface (arrowheads). Scale bar $=20 \mu \mathrm{m}$.

into its activity in a simple storage medium, the implant was maintained in physiological saline for an extended time periods. We found that it retained its microbiological activity and there was no change in its distribution over the implant surface (Antoci et al., 2008). In a more complex medium, serum, the antibiotic-modified surface preserved its biological activity even in the presence of plasma proteins. Indeed, as will be discussed later, in vivo studies indicate that that antibiotic activity is maintained in an animal model of bone repair when challenged with large numbers of bacteria. Whether there is a diminution in activity over time has not been determined. We also assessed the stability of the SAM to withstand disruptive forces such as those developed when implants are inserted into bone. We noted that the SAM layer remained stable following application of "press-pull" mechanical forces (Antoci et al., 2007c). The forces exerted on the implant (not measured) were configured to be in the same range as those that were used for the rat animal model study described later in this review. However, when a titanium screw was modified with antibiotic and then inserted into a mammalian bone sample, VAN antibody staining 
Fig. 3. Induction of infection at the osteotomy site in sheep. S. aureus was injected into the osteotomy gap through a silastic catheter. At time of closure of the soft tissue envelope, the catheter was placed next to the plate with the catheter tip at the level of the osteotomy site. Following completion of closure, the bacterial inoculum was carefully injected (see insert) and the catheter was removed prior to sealing the tissue portal with tissue glue.

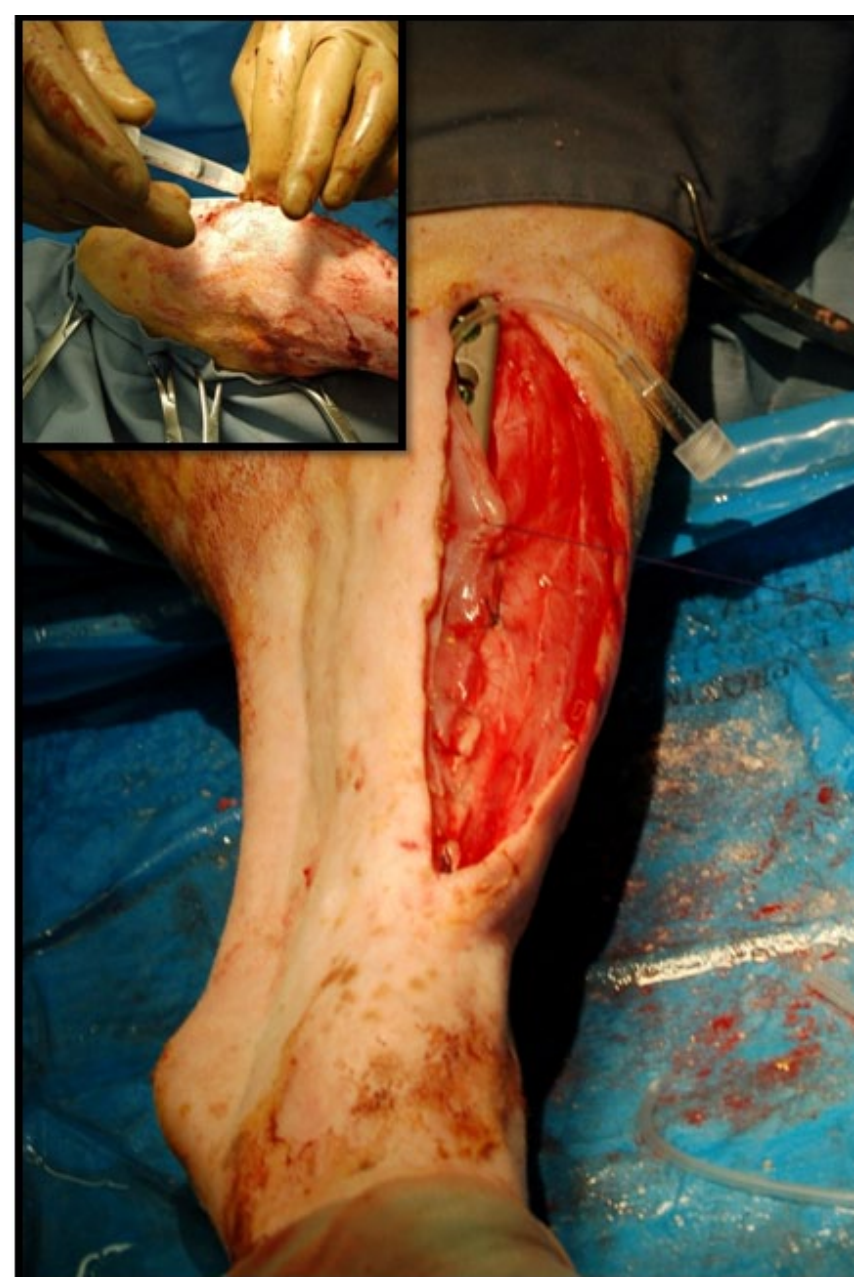

indicated an accumulation of antibiotic at the edges of the thread. Thus, while the VAN provided a stable, resistant and bioactive monolayer, torsional force caused its removal from the metal surface. Although there appears to be a physical limitation on the use of tethered antibiotics at sites of high torsion, the animal studies (below) indicate that for most surgeries the antibiotic is resistant to removal. Moreover, even at the high torsion sites, it would not be unreasonable to assume that the surface of the metal can be protected thereby preserving the integrity of the SAM and the activity of the antibiotic.

A wide range of microorganisms are present in the biofilm in a hip or knee implant. The most frequent infecting organisms are the coagulase negative staphylococci and $S$. aureus. To assess the antimicrobial activity of the tethered VAN, the surfaces were challenged with $S$. aureus, $S$. epidermidis as well as E. coli (Pulido et al., 2008). The Gram-positive $S$. aureus and Coagulase-negative $S$. epidermidis were chosen for study as these organisms are susceptible to VAN. E. coli is a Gram negative bacterium that is not killed by the antibiotic; it thus served as an internal negative control for the efficacy of the tethered antibiotic. In all cases, numbers of adherent bacteria and hence biocidal activity of the hybrid surface was determined by direct visualisation with the membrane permeable Syto9 dye (green) and the nuclear stain propidium iodide (red) (non-viable bacteria show some green and predominantly red staining whereas viable bacteria are green only). To quantitate biocidal activity, adherent bacteria were sonicated and following dilution and plating counted.

Fig. 2 shows that following incubation with $S$. epidermidis, the VAN-titanium surface fluorescence is very low for the period of the study $(30 \mathrm{~h})$ suggesting that there is minimal colonisation. In contrast, the unmodified surface (no VAN) is highly fluorescent, indicating that there is robust colonisation by the bacteria, a characteristic of biofilm formation. As expected, the tethered VAN was active against Gram-positive, but not Gram-negative organisms. Accordingly, when challenged with E. coli, colonisation was evident and of similar magnitudes on both the control (antibiotic-free) and the VAN-tethered surfaces (Antoci et al., 2008). All of these findings were quantitated by serial dilution and bacterial counting. Based on the results of this study, we concluded that the tethered antibiotic prevented bacterial adherence and biofilm formation with Gram-positive organisms, i.e., the organisms that are most commonly associated with periprosthetic infections.

\section{Influence of the tethered antibiotic on infected bone repair: animal models of bone infection}

There are very few small animal models of periprosthetic infection. Initially, we focused on using a rodent model as these animals are easy to maintain, cheap to house and resistant to systemic infections. However, while the 

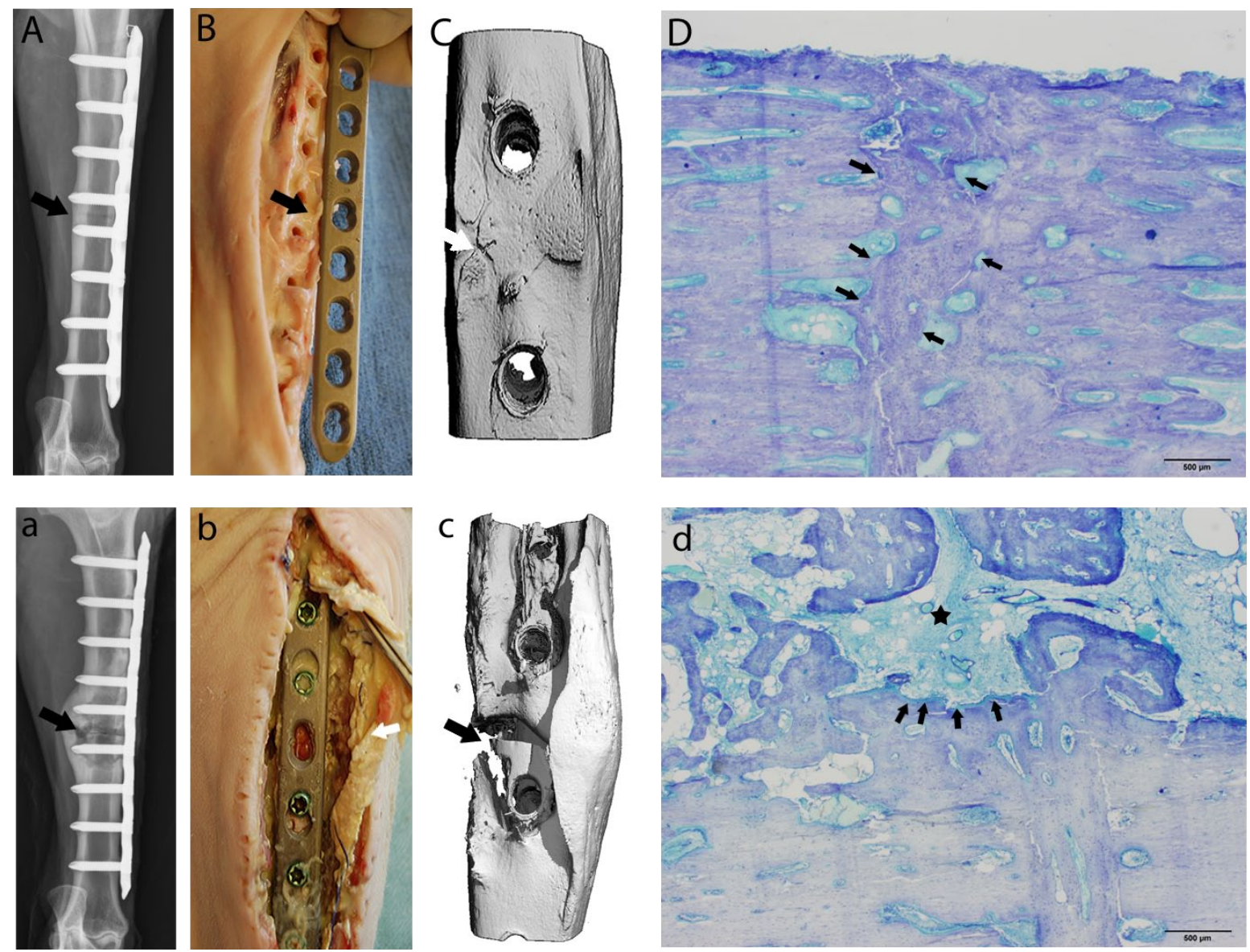

Fig. 4. Representative images at 90 days postoperative. (A-D) Tissue repaired with VAN-LCP (vancomycin-locking compression plate); (a-d): repair conducted with unaltered LCP): Digital radiographs with cranio-caudal view of osteotomy site confirming persistence of the osteotomy (a: arrow), and a healing osteotomy site (A: arrow). 3D reconstructed micro-CT views $(\mathbf{B b})$ of the osteotomy site correlating with digital radiographs Aa. Note the persistence of the osteotomy gap (arrows: a \& b). There was evidence of abscessation and an unstable osteotomy (c: arrow) during aseptic tissue harvest in the control cohort animals, while the tibiae repaired with coated plates presented with a grossly stable fracture callus and no evidence of infection $(\mathbf{C}$ : arrow). The histology images (Dd) corroborate the gross anatomic and radiographic findings. A representative toluidine blue-stained, mid-sagittal section from the treatment cohort. Scale bar $=500 \mu \mathrm{m}$. (D) comprising of the near cortex of the osteotomy site (row of arrows) is completely bridged by remodelling bone with minimal endosteal and periosteal callus formation. A photomicrograph (d) of a toluidine blue-stained, mid-sagittal section comprising the near cortex from sheep receiving an unaltered cpTi LCP demonstrates severe cortical lysis (arrows) with central cavity containing debris and pockets of suppurative inflammation with formation of large, poorly organised endosteal callus (asterisk). Scale bar $=500 \mu \mathrm{m}$.

rodent model has numerous advantages, a major limitation is its enhanced innate immuno-reactivity that provides a powerful barrier to bone infection. We overcame this drawback by infusing a known high concentration of bacteria directly into a long bone prior to inserting the VAN-modified titanium alloy pin. The concentration of infused organisms was many-fold higher than would be seen in the biofilm of an implant. Specifically, S. aureus (1.5 $\times 10^{3}$ colony forming units) was injected into the medullary cavities of rat femora after which the implant (an antibiotic tethered titanium alloy rod) was inserted into the canal. Signs of infection were evaluated weekly using radiography, and counts of bacteria adherent to the implant were assessed. These preliminary studies showed that the VAN-modified implant inhibited bacterial attachment and biofilm formation (Antoci et al., 2007d).

Based on the outcome of this study, we conducted a second investigation using a sheep model of infection. The design of this study was different from the rodent study in that the antibiotic was tethered to a locking plate used to promote bone healing in the face of a local infection. We performed a unilateral osteotomy in the mid-diaphyseal region of the tibia. To promote infection, $S$. aureus $(2.5$ $\mathrm{mL}, 10^{6}$ colony forming units $/ \mathrm{mL}$ ) was injected into the osteotomy gap through a silastic catheter after closure of all soft tissue layers, then the catheter was removed (see Fig. 3). While the bacterial load generated a florid local infection, organism number was adjusted such that it would overcome the animal's immune response at the operative site, without compromising the overall health of the animal. The osteotomy was reduced using a titanium locking compression plate in which the surface of the plate had been modified with VAN using the methodology described above. Again, it should be noted that the bacterial loading for this study was quite different from most human infections, where a relatively low number of 
microorganisms initiate biofilm formation. It is likely that the infection status was representative of an extreme case of osteomyelitis or PJI.

At the osteotomy site of the control sheep (standard locking plate, i.e., no VAN) the clinical signs were consistent with septic osteomyelitis, including delayed bony union and pain. In contrast, animals treated with the VAN locking plate exhibited fewer signs of postoperative pain when compared to control animals. These animals tended to be more ambulatory and less lame on their operated leg when evaluated on a conventional semiquantitative lameness scale of 1-4. However, there were no signs of systemic infection and the animals were bright and alert. By 90 days, gross-anatomic evaluation and CT analysis indicated that at the osteotomy site, the antibiotic tethered locking compression plate permitted remodelling: there was development of a bridging callus and bone healing, with minimal tissue discontinuity. The surgical wound had healed without any visible evidence of infection while radiographs confirmed that bone healing was progressing. In contrast, control animals displayed evidence of abscessation and an exuberant lytic callus, the medullary cavity was enlarged, there was periosteal lifting and evidence of mal- or incomplete union.

Histological analysis confirmed the clinical radiographic and ex vivo $\mathrm{CT}$ findings providing further insight into the bone changes (Fig. 4 A,a and C,c-D,d). The tethered antibiotic promoted formation of a bridging calcified callus with evidence of extensive remodelling. At some sites, in concert with the formation of new bone multinucleated osteoclasts were evident in resorption lacunae. In contrast, in sections of tissue from control animals, there was overall disorganisation of the primary callus and a lack of an endosteal callus. At the edges of the osteotomy, there was cortical and trabecular osteolysis. Necrotic neutrophils and other cells were seen in the remnants of the canalculi and Haversian canals of the bone (Fig. 4 D,d).

In terms of microbiology, at the time of implant removal, a bacterial culture was obtained at the level of the osteotomy site. In control animals, positive $S$. aureus cultures (3/4) were found in the callus region using standard clinical bacteriological methodologies: a pure growth of $S$. aureus was achieved in all cases. When compared with the treatment cohort, the microbiological results corroborated macroscopic and microscopic findings, namely $4 / 5$ animals yielded no bacterial growth from culture swabs.

\section{Summary and conclusion: from bench to bedside}

This short review provides a summary of previous work undertaken to generate a titanium implant that, through a display of covalently bonded antibiotics, provides a surface inhospitable to biofilm formation. Our general approach was to bond an antibiotic to aminopropylated titanium by solid phase peptide synthesis. We found that the tethered antibiotic was stable and active and because of the large number of surface molecules, the implant surface would be expected to limit development of resistant bacterial strains. Indeed, during the period of the study there was no emergence of resistance. In addition, we noted that the nanoscale surface was unaffected by push pull biomechanical usage of the type experienced in orthopaedic procedures.

The in vitro studies provided justification that despite the large number of synthetic steps in the generation of the covalent linkage, including the use of protective organic reagents, the antibiotic retained its activity. In the presence of the tethered VAN, there was a significant decrease in bacterial viability, number and adhesion. This finding is in marked contrast to the control surface that accumulated a considerable number of bacteria. When the latter surface was treated with crystal violet, a dye that stains exopolysaccharides there was evidence of biofilm formation on the untreated titanium (Antoci et al., 2008). Differences in the antimicrobial activities of the two surfaces were confirmed by microbiological counting, which also provided a quantitative estimate of the number of viable bacteria. In addition, SEM studies indicated the presence of an abundant, albeit poorly organised patches of thick biofilm and the presence of small colonies of bacteria on the control surface. Accordingly, the in vitro studies provided strong support for the notion that the titanium can be endowed with anti-bacterial activities by coupling an antibiotic directly to the metal surface. These studies begged the question: can the tethered antibiotic influence the course, severity and extent of an established bone infection?

When evaluated using both rodent and sheep animal models, there were the hallmark signs of bone infection without evidence of a marked systemic infection. The finding that there was normal bone healing in the absence of bacterial clustering lent considerable strength to the concept that the surface bound VAN was clinically efficient, not toxic to bone cells and protective. Of course, the sheep infection model was a better test of the implant to prevent a frank bacterial infection of bone rather than an assessment of infection associated with biofilm formation. Nevertheless, since the antibiotic killed large numbers of infecting organisms compared with the very small numbers that are associated with biofilm development, the findings lend strong support to our view that the system would be active in preventing the type of chronic infection linked to biofilm formation. Moreover, the study also clearly showed that the methodology that we have developed to tether antibiotics to titanium is adaptable to many types of surgical components that are used in clinical practice. Indeed, the possibility exists that by increasing the stability of the tethered antibiotic, the hybrid system could be used for almost all orthopaedic implant procedures. Based on the results of studies reviewed herein, we are of the opinion that this nano-engineered titanium surface is now ready for evaluation in a clinical environment for treatment of periprosthetic infections as well as difficult infections associated with osteomyelitis.

\section{Acknowledgements}

We thank the NIH (grants DE-13319, DE-10875, AR051303, DE019901, and HD061053), the Department of Defense (grant DAMD17-03-1-0713) and the Grayson 
Jockey Club Research Foundation for funding for this study.

Conflict of Interest: N.J. Hickok and J. Parvizi are part owners of SmartTech that has a commercial interest in the technology described in this manuscript.

\section{References}

Antoci V Jr, Adams CS, Hickok NJ, Shapiro IM, Parvizi J (2007a) Vancomycin bound to Ti rods reduces periprosthetic infection: preliminary study. Clin Orthop Relat Res 461: 88-95.

Antoci V Jr, Adams CS, Parvizi J, Ducheyne P, Shapiro IM, Hickok NJ (2007b) Covalently attached vancomycin provides a nanoscale antibacterial surface. Clin Orthop Relat Res 461: 81-87.

Antoci V Jr, King SB, Jose B, Parvizi J, Zeiger AR, Wickstrom E, Freeman TA, Composto RJ, Ducheyne P, Shapiro IM, Hickok NJ, Adams CS (2007c) Vancomycin covalently bonded to titanium alloy prevents bacterial colonization. J Orthop Res 25: 858-866.

Antoci V Jr, Adams CS, Hickok NJ, Shapiro IM, Parvizi J (2007d) Antibiotics for local delivery systems cause skeletal cell toxicity in vitro, Clin Orthop Relat Res 462: 200-206.

Antoci V Jr, Adams CS, Parvizi J, Davidson HM, Composto RJ, Freeman TA, Wickstrom E, Ducheyne P, Jungkind D, Shapiro IM, Hickok NJ (2008) The inhibition of Staphylococcus epidermidis biofilm formation by vancomycin-modified titanium alloy and implications for the treatment of periprosthetic infection. Biomaterials 29: 4684-4690.

Bahar G (2002) Biofilms, tolerance and antimicrobial resistance. Mikrobiyol Bul 36: 343-351.

Chen CP, Wickstrom E (2010) Self-protecting bactericidal titanium alloy surface formed by covalent bonding of daptomycin bisphosphonates. Bioconjug Chem 21: 1978-1986.

Cierny G, DiPasquale D (2002) Periprosthetic total joint infections: staging, treatment, and outcomes. Clin Orthop Relat Res 403: 23-28.

Costerton JW (1999) Bacterial biofilms: a common cause of persistent infections. Science 284: 1318-1322.

Danahy MP, Avaltroni MJ, Midwood KS, Schwarbauer JE, Schwartz J (2004) Self-assembled monolayers of alpha, omega-diphosphonic acids on Ti enable complete or spatially controlled surface derivatization. Langmuir 20: 5333-5337.

Donlan RM, Costerton JW (2002) Biofilms: survival mechanisms of clinically relevant microorganisms. Clin Microbiol Rev15: 167-193

Edupuganti OP, Antoci V Jr, King SB, Jose B, Adams CS, Parvizi J, Shapiro IM, Zeiger AR, Hickok NJ, Wickstrom E (2007) Covalent bonding of vancomycin to Ti6Al4V alloy pins provides long-term inhibition of Staphylococcus aureus colonization. Bioorg Med Chem Lett 17: 2692-2696.

El-Ghannam A, Jahed K, Govindaswami M (2010) Resorbable bioactive ceramic for treatment of bone infection. J Biomed Mater Res A 94: 308-316.
Fulkerson E, Valle CJ, Wise B, Walsh M, Preston C, Di Cesare PE (2005) Vancomycin-supplemented impacted bone allografts in infected hip arthroplasty. Two-stage revision results. Bone Joint Surg Br 87: 314-319

Geipel U, Herrmann M (2004) The infected implant. Part 1: Bacteriology. Orthopäde 33: 1411-1428.

Grimsrud C, Raven R, Fothergill AW, Kim HT (2011) The in vitro elution characteristics of antifungal-loaded PMMA bone cement and calcium sulfate bone substitute. Orthopedics 34: e378-81.

Hanssen AD (2004) Prophylactic use of antibiotic bone cement: an emerging standard - in opposition. J Arthroplasty 19: 73-77.

Hickok NJ, Ketonis C, Adams CS (2011) Tethered antibiotics. In: Ducheyne P, Healy KE, Hutmacher DW, Grainger DW, Kirkpatrick CJ (eds) Comprehensive Biomaterials, vol. 4, Elsevier, Amsterdam. pp. 281-294.

Jose B, Antoci V Jr, Zeiger AR,Wickstrom E, Hickok NJ (2005) Vancomycin covalently bonded to titanium beads kills Staphylococcus aureus. Chem Biol 12: 10411048.

Kahne D, Leimkuhler C, Lu W, Walsh C (2005) Glycopeptide and lipoglycopeptide antibiotics. Chem Rev 105: 425-428.

Ketonis C, Parvizi J, Adams CS, Shapiro IM, Hickok NJ (2009) Topographic features retained after antibiotic modification of Ti alloy surfaces: retention oftopography with attachment of antibiotics Clin Orthop Relat Res 467: 1678-1687.

Ketonis C, Adams CS, Barr S, Aiyer A, Shapiro IM, Parvizi J, Hickok NJ (2010) Antibiotic modification of native grafts: improving upon nature's scaffolds, Tissue Eng A 16: 2041-2049.

Khoo X, Hamilton P, O’Toole GA, Snyder BD, Kenan DJ, Grinstaff MW (2009) Directed assembly of PEGylatedpeptide coatings for infection-resistant titanium metal. J Am Chem Soc 131: 10992-10997.

Lawson MC, Bowman CN, Anseth KS (2007) Vancomycin derivative photopolymerized to titanium kills S. epidermidis, Clin Orthop Relat Res 461: 96-105.

Lee MH, Brass DA, Morris R, Composto RJ, Ducheyne $\mathrm{P}$ (2005) The effect of non-specific interactions on cellular adhesion using model surfaces. Biomaterials 26: 17211730 .

Loll PJ, Axelsen PH (2000) The structural biology of molecular recognition by vancomycin. Annu Rev Biophys Biomol Struct 29: 265-289.

McPherson EJ, Woodson C, Holtom P, Roidis N, Shufelt C, Patzakis MJ (2002) Periprosthetic total hip infection: outcomes using a staging system. Clin Orthop Relat Res 403: 8-15.

Mikulec LJ, Puleo DA (1996) Use of p-nitrophenyl chloroformate chemistry to immobilize protein on orthopedic biomaterials. J Biomed Mat Res 32: 203-208.

Moojen DJ, Hentenaar B, Charles Vogely H, Verbout AJ, Castelein RM, Dhert WJ (2008) In vitro release of antibiotics from commercial PMMA beads and articulating hip spacers. J Arthroplasty 23: 1152-1156.

Puleo DA, Kissling RA, Sheu MS (2002) A technique to immobilize bioactive proteins, including bone 
morphogenetic protein-4 (BMP-4) on titanium alloy. Biomaterials 23: 2079-2087.

Pulido L, Ghanem E, Joshi A, Purtill JJ, Parvizi J (2008) Periprosthetic joint infection: the incidence, timing, and predisposing factors. Clin Orthop Relat Res 466: 17101715.

Secchi AG, Grigoriou V, Shapiro IM, Cavalcanti-Adam EA, Composto RJ, Ducheyne P, Adams CS (2007) RGDS peptides immobilized on titanium alloy stimulate bone cell attachment, differentiation and confer resistance to apoptosis, J Biomed Mater Res A 83: 577-584.

Stewart PS, Costerton JW (2001) Antibiotic resistance of bacteria in biofilms. Lancet 358: 135-138.

Toms AD, Davidson D, Masri BA, Duncan CP (2006) The management of peri-prosthetic infection in total joint arthroplasty. J Bone Joint Surg Br 88: 149-155.

Watts AE, Nixon AJ, Papich MG, Sparks HD, Schwark WS. (2011) In vitro elution of amikacin and ticarcillin from a resorbable, self-setting, fiber reinforced calcium phosphate cement. Vet Surg 40: 563-570.

Wojcik M, Puleo DA (1997) Biochemical surface modification of Ti-6Al-4V for the delivery of protein to the cell-biomaterial interface. Biomed Sci Instrum 33: 166-171.

\section{Discussion with Reviewers}

Reviewer I: The authors are obviously heavily invested in this technology with what seems to be a start-up company having spun-off from this body of work. Do they feel another lab would obtain the same results and is there a plan for corroborating evidence by sharing this proprietary information?

Authors: The reviewer is correct in that a start-up company, SmartTech was formed to pursue IP protection after the university decided to release the technology; Dr. Hickok has a conflict because of this start-up. We have performed these syntheses in more than one laboratory. Within Jefferson, both the Wickstrom lab and the Hickok lab have successfully performed these syntheses, with personnel changing over the years as evidenced by the publication lists. We had mixed results when one other lab to date attempted to reproduce the work - these mixed results were attributed to issues with the solubility and activity of the parent vancomycin.

Specifically, the chemistry is based on the solid-state peptide synthesis reaction scheme that is used in every commercial peptide synthesiser. Based on unpublished results, we think that the organic reagents used for the coupling denatures some of the vancomycin, the percentage of which depends on the source. It should be noted that there is no "proprietary" procedure for the synthesis - our published papers describe the complete reaction scheme for production of these surfaces. It should be noted that K.S. Anseth at the University of Colorado has used a similar reaction scheme to immobilise polymerised vancomycin to surfaces and also finds that these derivatised surfaces prevent bacterial colonisation (Lawson et al., 2007, text reference; Lawson et al., 2009; Lawson et al., 2010, additional references). Therefore, while there is clearly a conflict associated with Dr. Hickok's role in SmartTech, we are confident that this conflict has not affected our interpretation of the in vitro and in vivo studies and that a skilled chemist can synthesise and test these surfaces based on the descriptions that we and others have reported elsewhere.

Reviewer II: The prophylaxis and treatment of infection are two separate scenarios. In the manuscript you indicate the potential of the surface to treat infection and the sheep study proves its effectiveness in the prevention of infection. Do you think this coating is more suitable for treatment or prophylaxis of infection, or both?

Authors: The major role of the tethered antibiotic layer is to prevent the formation of a biofilm. If a biofilm is established on an implant, then the surgeon has a very difficult problem in that it harbours organisms that populate not just the implant surface, but also bone interstices. To eradicate the infection, prior to inserting a new implant, high concentrations of powerful antibiotics are necessitated together with rigorous debridement techniques. From this perspective, if arthroplasty is to be successful, then prevention of an infection is mandated. Based on the pre-clinical studies described in this review, the tethered antibiotic would provide a powerful barrier against implant colonisation and biofilm generation. From a practical viewpoint, probably the best use of the implant would be in those circumstances where there is a propensity for infection. For example, for those patients who are immunocompromised or immunodeficient. For these individuals, the implant would serve a prophylactic role.

\section{Additional References}

Lawson MC, Shoemaker R, Hoth KB, Bowman CN, Anseth KS (2009) Polymerizable vancomycin derivatives for bactericidal biomaterial surface modification: structurefunction evaluation. Biomacromolecules 108: 2221-2234.

Lawson MC, Hoth KC, Deforest CA, Bowman CN, Anseth KS (2010) Inhibition of Staphylococcus epidermidis biofilms using polymerizable vancomycin derivatives. Clin Orthop Rel Res 468: 2081-2091. 